\title{
Designing adiabatic time evolution from high-frequency bichromatic sources
}

\author{
Álvaro Gómez-León $\odot$ and Gloria Platero $\odot$ \\ Instituto de Ciencia de Materiales de Madrid (CSIC), E-28049 Madrid, Spain
}

(Received 25 February 2020; accepted 24 August 2020; published 15 September 2020)

\begin{abstract}
We investigate the quantum dynamics of a two-level system driven by a bichromatic field, using a nonperturbative analysis. We make special emphasis in the case of two large frequencies, where the Magnus expansion can fail, and in the case of a large and a small frequency, where resonances can dominate. In the first case, we show that two large frequencies can be combined to produce an effective adiabatic evolution. In the second case, we show that high-frequency terms (which naturally arise as corrections to the adiabatic evolution obtained in the first case) can be used to produce a highly tunable adiabatic evolution over the whole Bloch sphere, controlled by multiphoton resonances.
\end{abstract}

DOI: 10.1103/PhysRevResearch.2.033412

\section{INTRODUCTION}

Perturbing a system out of equilibrium is at the heart of physics, as it allows one to extract information about its properties by just measuring the response to the perturbation. Besides small perturbations, one can also produce nonlinear effects of high complexity, and steady states with novel properties such as Floquet topological insulators, skyrmions, or time crystals [1-6]. Applying periodic perturbations has been shown to be a versatile tool to manipulate physical systems. For instance, they allow one to control spin qubits in quantum dots [7-11], or to induce new electronic, dynamical, and topological properties [12-16]. These works typically consider monochromatic driving, although bichromatic fields have been used in a few occasions [17-20], showing that their potential has not been fully explored.

The periodically driven two-level system is one of the fundamental models in quantum mechanics. Its physical realization has been successfully implemented in quantum dots [21-24], complex molecules [25,26], superconducting devices [27], and many other systems [28]. Its universality relies on the fact that many quantum mechanical systems, when truncated to their low-lying states by lowering the temperature, can reduce to the dynamics between the ground state and the first excited state.

The Hamiltonian describing the unperturbed two-level system usually displays a splitting $\Delta_{z}$. Then, one chooses this direction as the quantization axis, and performs transitions between the ground state and the excited state to probe the system, which is the guiding principle in techniques such as nuclear and electron spin resonance. The Hamiltonian describing the model can be written as $H(t)=H_{0}+V(t)$,

Published by the American Physical Society under the terms of the Creative Commons Attribution 4.0 International license. Further distribution of this work must maintain attribution to the author(s) and the published article's title, journal citation, and DOI. with

$$
\begin{aligned}
H_{0} & =\frac{\Delta_{z}}{2} \sigma_{z}, \\
V(t) & =\sum_{i} \frac{V_{i}}{2} f_{i}\left(t, \omega_{i}, \phi_{i}\right) \sigma_{x},
\end{aligned}
$$

and where $V_{i}, \omega_{i}$ and $\phi_{i}$ correspond to the different amplitudes, frequencies, and phases of the external source, respectively. The dynamics of this simple Hamiltonian can be complicated, even in the monochromatic case, as the three energy scales involved $\left(\Delta_{z}, V_{1}\right.$, and $\left.\omega_{1}\right)$ can lead to very different behavior. The standard perturbative analysis in $V_{1} \ll \Delta_{z}, \omega_{1}$ explains the linear response regime. This is commonly used to probe the system and obtain information about its physical properties [29]. On the other hand, one can consider the (high-frequency) strongly driven regime $V_{1}>\omega_{1}$ and $\omega_{1} \gg$ $\Delta_{z}$, which produces the spectral changes typically found in Magnus expansions [30], and can be used to dynamically tune the properties of the system [2,5,12,31,32]. Finally, the resonant behavior corresponds to the case $\omega_{1} \simeq \Delta_{z}$, which produces a transfer of spectral weight from the ground state to the excited state. This is used for state preparation in many experiments [33] or to induce single-qubit gates [24]. On top of that, environmental degrees of freedom in experiments can also couple to the external field, producing an undesired large signal if they are resonant.

In this work we study a two-level system driven by a bichromatic field and discuss the advantages over the monochromatic case. We analyze the limitations of highfrequency expansions, when more than one frequency is present, and introduce a description which captures the full dynamics, including the fine details of the micro-motion. This is in contrast with expansions where just the stroboscopic evolution is considered, missing the dynamics within a period, which can be crucial for a topological analysis [34].

We find that when the two frequencies are large, but close to each other, the field amplitudes can be controlled to produce an effective adiabatic evolution (see Fig. 1). We demonstrate 


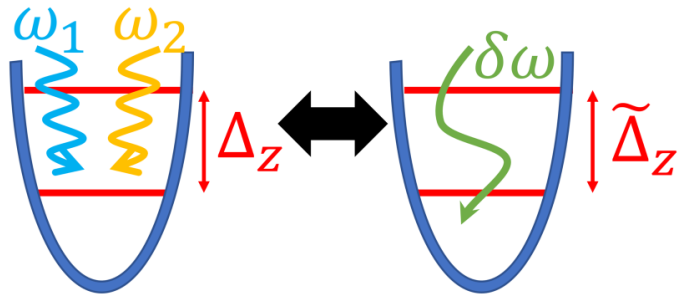

FIG. 1. Schematic representation where a system driven by a high-frequency bichromatic field behaves as adiabatically driven by a single frequency.

that the adiabatic behavior is robust to noise, and highly tunable. Furthermore, we show that nonadiabatic corrections to the resulting effective Hamiltonian can be beneficial, and can be used to engineer adiabatic rotations on the Bloch sphere. We also demonstrate that the long-time dynamics is controlled by multiphoton resonances. In addition, our analysis can be extended to the analysis of a multilevel system under multichromatic driving in a straightforward manner.

\section{MOTIVATION}

As an illustrative example to understand the breakdown of high-frequency expansions in a multifrequency case, let us first consider a rather simple trigonometric property of the function $g(t)=\cos (\omega t)$. It can generally be written as

$$
g(t)=\cos \left(\omega_{1} t\right) \cos \left(\omega_{2} t\right)+\sin \left(\omega_{1} t\right) \sin \left(\omega_{2} t\right),
$$

where $\omega=\omega_{1}-\omega_{2}$ and their sum is arbitrary. If $\omega_{i} \gg \omega$, one can interpret the slowly evolving oscillatory function $g(t)$ as coming from the difference of two large frequencies, whose difference is very small. In this case, what makes it possible to exactly map the two high frequencies to an adiabatic evolution is the specific relation between their Fourier components, where only the crossed terms $g_{ \pm 1, \mp 1}$ of the two-dimensional Fourier expansion $g_{n_{1}, n_{2}}=$ $\int_{0}^{2 \pi} \frac{d \theta_{1}}{2 \pi} \int_{0}^{2 \pi} \frac{d \theta_{2}}{2 \pi} e^{-i\left(n_{1} \theta_{1}+n_{2} \theta_{2}\right)} g_{\vec{\theta}}$ contribute, with $g_{\vec{\theta}}$ being the function $g(t)$ reparametrized according to $\theta_{i}=\omega_{i} t$ [35]. This illustrates a specific case where a system driven by two initially large frequencies will not give a converging result using a high-frequency expansion. The reason is that a system with such a driving term (with $\omega$ smaller than all the characteristic energies of the model), obviously requires an adiabatic analysis [36] due to its slow time evolution. Furthermore, it provides some intuition about the requirements to engineer a specific dynamical behavior in a quantum system, by studying its Fourier decomposition.

\section{BICHROMATIC TWO-LEVEL SYSTEM}

Let us now move to the problem at hand. We choose a simple harmonic protocol for the external drive $f_{i}\left(t, \omega_{i}, \phi_{i}\right)=$ $\cos \left(\omega_{i} t+\phi_{i}\right)$, although other choices are possible ( $i$ labels each different component of the drive). One could also choose each term in Eq. (2) coupled to a different, noncommuting, degree of freedom (e.g., to a $\sigma_{y}$ component). However, this is not necessary for the present analysis and will be discussed below. We consider the specific case of bichromatic drive, where the sum in Eq. (2) is restricted to two terms only. Each term is characterized by a different amplitude, frequency, and phase. If we parametrize $\omega_{i} t \rightarrow \theta_{i}$ to obtain a two-dimensional Fourier representation [35], the only nonvanishing Fourier components are the terms $H_{0, \pm 1}$ and $H_{ \pm 1,0}$, which characterize the time-evolution operator for small field amplitudes. This indicates that for weak amplitudes, the behavior is dominated by oscillations with frequencies $\omega_{1,2}$ only.

If instead we perform a nonperturbative analysis of $H(t)$, by applying the transformation $\mathcal{U}(t)=\exp \left\{-i \int V(t) d t\right\}$ we find the following transformed multichromatic timedependent Hamiltonian:

$$
\begin{aligned}
\tilde{H}(t)= & \frac{\Delta_{z}}{4}\left(\sigma_{z}-i \sigma_{y}\right) \prod_{i} e^{i F_{i}\left(t, \omega_{i}, \phi_{i}\right)} \\
& +\frac{\Delta_{z}}{4}\left(\sigma_{z}+i \sigma_{y}\right) \prod_{i} e^{-i F_{i}\left(t, \omega_{i}, \phi_{i}\right)},
\end{aligned}
$$

where we have defined $F_{i}\left(t, \omega_{i}, \phi_{i}\right)=\int V_{i} f_{i}\left(t, \omega_{i}, \phi_{i}\right) d t$, where this result is still valid for an arbitrary number of drive components. The advantage of Eq. (4) relies on the fact that, from a Jacobi-Anger expansion [37], one finds nonperturbative expressions in $V_{i}$. Notice that this type of Hamiltonian is directly obtained in electronic systems via the Peierls substitution [12], indicating that our results will be valid for seemingly different systems, connected by unitary transformations. However, in the case of the Peierls substitution, the transformation to the interaction picture is not required.

In the monochromatic case, the phase $\phi_{1}$ in Eq. (4) does not affect the spectrum, because it is a gauge degree of freedom that sets the origin of the time evolution, but it affects the dynamics. However, in the multichromatic case the phase differences are relevant and both, the spectrum and the dynamics are affected. This provides an extra degree of freedom in Floquet engineering, absent in the monochromatic case, although we will not make use of it in the present work.

The two-dimensional Fourier decomposition of Eq. (4) (we parametrize $\left.\theta_{i}=\omega_{i} t\right)$,

$$
\tilde{H}_{n_{1}, n_{2}}=\int_{0}^{2 \pi} \frac{d \theta_{1}}{2 \pi} \int_{0}^{2 \pi} \frac{d \theta_{2}}{2 \pi} \tilde{H}\left(\theta_{1}, \theta_{2}\right) e^{-i\left(n_{1} \theta_{1}+n_{2} \theta_{2}\right)},
$$

leads to the following expression for the Fourier components of the Hamiltonian:

$$
\begin{aligned}
\tilde{H}_{n_{1}, n_{2}}= & \frac{\Delta_{z}}{4} e^{i\left(n_{1} \phi_{1}+n_{2} \phi_{2}\right)} J_{n_{1}}\left(\alpha_{1}\right) J_{n_{2}}\left(\alpha_{2}\right)\left(\sigma_{z}-i \sigma_{y}\right) \\
& +\frac{\Delta_{z}}{4} e^{i\left(n_{1} \phi_{1}+n_{2} \phi_{2}\right)} J_{-n_{1}}\left(\alpha_{1}\right) J_{-n_{2}}\left(\alpha_{2}\right)\left(\sigma_{z}+i \sigma_{y}\right)
\end{aligned}
$$

where $\alpha_{i}=V_{i} / \omega_{i}$ and we have used the Jacobi-Anger expan$\operatorname{sion} e^{i z \sin (\theta)}=\sum_{n=-\infty}^{\infty} J_{n}(z) e^{i n \theta}$. Equation (6) can be further simplified if the two frequencies are commensurate [14,37]; however, we will consider the general case.

Notice that Eq. (6) contains an infinite number of Fourier components, and, in contrast with the unrealistic, but pedagogical case of $g(t)$ above, the adiabatic behavior will only happen if we can enhance the crossed Fourier components $\tilde{H}_{ \pm 1, \mp 1}$. Fortunately, due to the nonperturbative expressions in $\alpha_{i}$ this is now possible, and we choose values of $\alpha_{i}$ that maximize $J_{1}\left(\alpha_{i}\right)$, while requiring $\omega_{-} \ll \Delta_{z}$ and $\omega_{i} \gg \Delta_{z}$ (we 


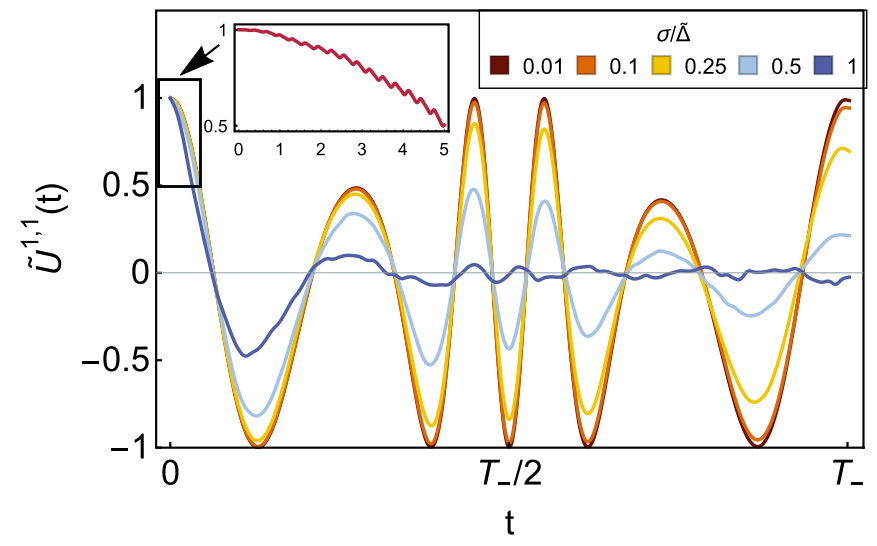

FIG. 2. Exact dynamics during an adiabatic period $T_{-}=2 \pi / \omega_{-}$ for the real part of the upper diagonal element of the time-evolution operator $\tilde{U}(t)$. The presence of white noise following a normal distribution with a standard deviation $\sigma$ has been considered. The colors indicate different noise strength $\sigma$, in units of the dominant energy scale $\tilde{\Delta}=\Delta_{z} J_{1}^{2}(\alpha)$. Parameters: $\omega_{1} / \Delta_{z}=10, \omega_{2} / \Delta_{z}=10.05$, $\alpha \simeq 1.8$ [first maximum of $J_{1}(\alpha)$ ], and $\phi_{i}=0$. The inset shows the short time dynamics, where the fast oscillations from the fast applied drive (with period $T_{1,2} \simeq 2 \pi / \omega_{1,2}$ ) are more evident. All the other components of the evolution operator also evolve adiabatically.

have defined $\omega_{ \pm}=\omega_{1} \pm \omega_{2}$ and fixed $\phi_{i}=0$ for simplicity):

$$
\begin{aligned}
\tilde{H}(t) \simeq & \Delta_{z}\left[\frac{J_{0}^{2}(\alpha)}{2}-J_{1}^{2}(\alpha) \cos \left(\omega_{-} t\right)\right] \sigma_{z} \\
& +\Delta_{z} J_{1}^{2}(\alpha) \cos \left(\omega_{+} t\right) \sigma_{z} \\
& +\Delta_{z} J_{0}(\alpha) J_{1}(\alpha) \sum_{i=1,2} \sin \left(\omega_{i} t\right) \sigma_{y}+\cdots
\end{aligned}
$$

Noticing that the fast oscillating term in the second line averages to zero in this regime, and that the third line contributes with a small amplitude correction to the time evolution, we obtain the next leading Hamiltonian:

$$
\tilde{H}_{0}(t) \simeq \Delta_{z}\left[\frac{J_{0}^{2}(\alpha)}{2}-J_{1}^{2}(\alpha) \cos \left(\omega_{-} t\right)\right] \sigma_{z}
$$

Actually as $\omega_{i} \gg \Delta_{z}$, all terms with a fast frequency dependence, of the order of $\omega_{i}$ or larger, tend to zero as $\omega_{i} \rightarrow \infty$. To demonstrate this we have numerically calculated the time evolution operator $\tilde{U}(t)$ in the interaction picture. Figure 2 shows the real part of the component $\tilde{U}^{1,1}(t)$ over time, which is related with the occupation probability of the excited state. Different colors indicate the time evolution averaged over a hundred realizations of white noise, following a normal distribution with standard deviation $\sigma$. For weak noise, the plot displays adiabatic behavior (notice that $T_{-} \sim 125$ while $T_{1,2} \sim 0.6$ is the period of the driving fields) with small and fast amplitude oscillations around the mean value, coming from the high-frequency corrections (see Fig. 2, inset). This is one of the main results of this work: One can combine two high-frequency drives to effectively evolve the system adiabatically. Actually, Fig. 2 contains a combination of two different periods, which can be externally controlled: harmonic oscillations due to the static part of the effective Hamiltonian [first term in Eq. (8)], and a slow frequency modulation due to the adiabatic term [second term in Eq. (8)]. Reducing the difference $\omega_{-}=\omega_{1}-\omega_{2}$ makes the static part dominate at short times, while its increase makes the nonlinear, adiabatic term to take over (these two cases are explicitly shown in Appendix A, Fig. 4 with an extended discussion about the role of each term in the effective time-dependent Hamiltonian).

In addition, the robustness of our prediction is illustrated with the changes of the time evolution as the noise increases. Figure 2 shows that the original behavior persists for weak values of noise, until $\sigma$ becomes of the order of the dominant energy scale, where the oscillations are strongly damped. This indicates that experiments would have time to perform a few adiabatic cycles before the effect of noise takes over.

\section{CORRECTIONS TO THE EFFECTIVE ADIABATIC HAMILTONIAN}

We have shown that it is possible to drive a system with two different frequencies and produce effective adiabatic behavior. The dominant part of the Hamiltonian, shown in Eq. (8), characterizes the slow evolution, but corrections due to oscillatory terms with higher frequency are also present and they can produce transitions between the adiabatic eigenstates [specially relevant are the terms proportional to $\sigma_{y}$ in Eq. (7), because they do not commute with the leading order Hamiltonian]. To understand their effect we now introduce a general formalism to study Hamiltonians with two different frequencies, where each drive couples to a different degree of freedom. The general form of the Hamiltonian can be compactly written as

$$
\tilde{H}(t)=\tilde{H}_{0}(t)+\epsilon \tilde{H}_{1}(t),
$$

where

$$
\begin{aligned}
& \tilde{H}_{0}(t)=\frac{\tilde{\Delta}(t)}{2} \sigma_{z}, \\
& \tilde{H}_{1}(t)=\frac{\tilde{V}(t)}{2} \sigma_{y} ;
\end{aligned}
$$

the functions $\tilde{\Delta}(t)$ and $\tilde{V}(t)$ are, for the moment, general harmonic functions, and $\epsilon$ is introduced to organize the perturbative series (we take $\epsilon \rightarrow 1$ at the end of the calculations). To study the nonperturbative dynamics we will calculate the time-evolution operator using multiple-scales analysis. This method deals with the fastest timescales first, and then each correction characterizes new processes taking over at longer times. Importantly, this method includes a renormalization procedure for the secular terms [38-40], making the solutions valid at high or low frequency, as well as near a resonance. This approach only neglects processes that take over at longer timescales than the order of the expansion in $\epsilon$.

In the first step we parametrize the time evolution operator $U(t) \rightarrow U(\vec{\tau})$ in terms of a set of timescales $\tau_{n}=\epsilon^{n} t$, and expand in powers of $\epsilon$ the equation of motion for the timeevolution operator, with $U(\vec{\tau})=\sum_{n} \epsilon^{n} U_{n}(\vec{\tau})$. The equations to zeroth and first order in $\epsilon$ result in

$$
\begin{gathered}
i \partial_{\tau_{0}} U_{0}=\tilde{H}_{0}\left(\tau_{0}\right) U_{0}, \\
i \partial_{\tau_{0}} U_{1}+i \partial_{\tau_{1}} U_{0}=\tilde{H}_{0}\left(\tau_{0}\right) U_{1}+\tilde{H}_{1}\left(\tau_{0}\right) U_{0},
\end{gathered}
$$


where we have omitted the $\vec{\tau}$ dependence in $U_{n}(\vec{\tau})$. The lowest order solution can be easily obtained from Eq. (11) by direct matrix exponentiation:

$$
U_{0}(\vec{\tau})=e^{-i \int_{0}^{\tau_{0}} \tilde{H}_{0}\left(\tau_{0}\right) d \tau_{0}} u_{0}\left(\tau_{1}\right),
$$

where the matrix $u_{0}\left(\tau_{1}\right)$ comes from the boundary condition and will be determined later on, during the renormalization procedure. Equation (13) can now be inserted in Eq. (12) and solved by choosing $U_{1}(\vec{\tau})=u_{1}(\vec{\tau}) v_{1}(\vec{\tau})$, with $u_{1}(\vec{\tau})=$ $e^{-i \int_{0}^{\tau_{0}} \tilde{H}_{0}\left(\tau_{0}\right) d \tau_{0}}$ being the solution to the homogeneous equation. The solution results in

$$
\begin{aligned}
U_{1}(\vec{\tau})= & -i u_{1}\left(\tau_{0}\right) \int_{0}^{\tau_{0}} \tilde{H}_{1}^{\prime}\left(\tau_{0}\right) d \tau_{0} u_{0}\left(\tau_{1}\right) \\
& -\tau_{0} u_{1}\left(\tau_{0}\right) \partial_{\tau_{1}} u_{0}\left(\tau_{1}\right),
\end{aligned}
$$

where we have defined

$$
\tilde{H}_{1}^{\prime}\left(\tau_{0}\right)=u_{1}\left(\tau_{0}\right)^{-1} \tilde{H}_{1}\left(\tau_{0}\right) u_{1}\left(\tau_{0}\right) .
$$

Equations (13) and (14) are the formal solutions for the time evolution operator, which now need to be particularized for the case of interest and renormalized, if needed. For our present purpose we fix the specific form of the periodic functions to $\tilde{\Delta}(t)=\tilde{\Delta}_{z}+\mu \cos (\omega t)$ and $\tilde{V}(t)=\beta \cos (\Omega t)$. In this case $\tilde{\Delta}_{z}$ corresponds to the static part [first term in Eq. (8)], $\mu$ to the dominant time-dependent term [second term in Eq. (8)], and $\beta$ to the dominant correction, noncommuting with Eq. (8) (the value of the two frequencies $\omega$ and $\Omega$ is arbitrary for the moment). The unperturbed solution is obtained from Eq. (13) and displays the nonlinear phase evolution typically obtained in time-dependent systems:

$$
U_{0}(\vec{\tau})=e^{-\frac{i}{2}\left[\tilde{\Delta}_{z} \tau_{0}+\frac{\mu}{\omega} \sin \left(\omega \tau_{0}\right)\right] \sigma_{z}} u_{0}\left(\tau_{1}\right),
$$

where $u_{0}\left(\tau_{1}\right)$ still needs to be determined. Similarly, the firstorder solution in $\epsilon$ is obtained from Eq. (14). We do not write here the full form of the solution, because of its length and because it is enough to show that it is proportional to (details of the calculation are in Appendix B)

$$
U_{1}(\vec{\tau}) \propto \beta\left[\left(n \omega \pm \tilde{\Delta}_{z}\right)^{2}-\Omega^{2}\right]^{-1} .
$$

This correction to $U_{0}(\vec{\tau})$ diverges if the denominator in Eq. (17) vanishes. This is a common feature of timedependent perturbation theory, indicating the breakdown of the solution, but these resonances can be renormalized in multiple-scales analysis, and produce nonperturbative corrections to $U_{0}(\vec{\tau})$. Strictly speaking, the resonance condition can only be fulfilled for commensurate frequencies (which differentiates this case with the one of incommensurate frequencies), and for very specific values of the parameters. However, if the denominator in Eq. (17) becomes smaller than $\beta$, the perturbative series still diverges and should be renormalized as well, making the difference between incommensurate and commensurate frequencies (with very long total period) merely a mathematical curiosity, for the physically relevant timescales of this setup. Therefore, one can relax the strict relation between the parameters for a resonance, to just the approximate one: $\left(n \omega \pm \tilde{\Delta}_{z}\right)^{2}-\Omega^{2} \lesssim \beta$.

To renormalize the resonant terms, one needs to separate resonant and off-resonant contributions. The amplitude corrections produced by the off-resonant terms in Eq. (17) are of order $\beta$, and can be neglected if we focus on $U_{0}(t)$ only. However, resonant corrections contribute to leading order and need to be included. We assume that the system is in the regime $\omega \ll \tilde{\Delta}_{z} \ll \Omega$, where $\omega$ corresponds to an adiabatic drive and $\Omega$ to a high-frequency one. This situation is analogous to the one obtained in Eq. (7) for the effective adiabatic Hamiltonian. In this situation, several resonances can contribute (i.e., several values of $n$ fulfill $\left(n \omega \pm \tilde{\Delta}_{z}\right)^{2}-\Omega^{2} \lesssim \beta$ ), while in a different regime the analysis would be simpler, because the resonances do not need to be included. Then, in the spirit of multiple-scales analysis, we require that the secular terms produced by the resonances are canceled by $\partial_{\tau_{1}} u_{0}\left(\tau_{1}\right)$ in Eq. (14). This requirement leads to the following flow equation for $u_{0}\left(\tau_{1}\right)$ :

$$
\partial_{\tau_{1}} u_{0}\left(\tau_{1}\right)=-i \frac{\beta}{4} \sum_{n_{0}} J_{n_{0}}\left(\frac{\mu}{\omega}\right) \sigma_{y} u_{0}\left(\tau_{1}\right)
$$

where $n_{0}$ corresponds to the set of resonances $\left\{ \pm n_{0}\right\}$ which fulfill the approximate resonance condition above. This equation allows one to determine $u_{0}\left(\tau_{1}\right)$, which encodes the nonperturbative correction to $U_{0}(t)$. The lowest order renormalized solution becomes

$$
U_{0}(t) \simeq e^{-\frac{i}{2}\left[\tilde{\Delta}_{z} t+\frac{\mu}{\omega} \sin (\omega t)\right] \sigma_{z}} e^{-i t \frac{\beta}{4} \sum_{n_{0}} J_{n_{0}}\left(\frac{\mu}{\omega}\right) \sigma_{y}} .
$$

Notice that the smaller $\omega$ is, the larger is the set of resonances $\pm n_{0}$ that needs to be included, increasing the contribution from the nonperturbative correction. Furthermore, this correction strongly depends on the ratio $\mu / \omega$. This indicates that if the system is far from a resonance, or $\mu$ is not in the region where $J_{n_{0}}(\mu / \omega)$ has a relevant weight, the behavior is similar to that of the unperturbed solution (at least to timescales of the order of $\beta^{-2}$ ).

The multiple-scales analysis can be continued to higher orders in a very systematic way; however, the results presented here are quite accurate for the range of parameters under consideration. In Fig. 3 we show a comparison between the numerical and the analytical approximation for the timeevolution operator. One can identify three different timescales in this plot:

(1) The shortest timescale is given by slow harmonic oscillations coming from the static part of the unperturbed solution $\tilde{\Delta}_{z}$ (as those oscillating between \pm 1 in Fig. 2).

(2) The next timescale corresponds to the nonlinear phase evolution. Proportional to $\mu$, it introduces the anharmonic oscillations happening at intermediate times and defines the adiabatic period $T_{-}$.

(3) The longest timescale is produced by the nonperturbative correction produced by $\tilde{H}_{1}(t)$. It produces the long-time modulation observed in Fig. 3 (in this case $T_{-} \sim 60$ and the long-time modulation has period $\tau_{\text {long }} \sim 600$, i.e., one order of magnitude larger).

We also show the dynamics for the off-diagonal component of $U(t)$ in the Appendix B, Fig. 7, to confirm that the long-time behavior is controlled by the nonperturbative correction $u_{1}\left(\tau_{1}\right)$, which produces the rotation proportional to $\sigma_{y}$ in Eq. (19). 


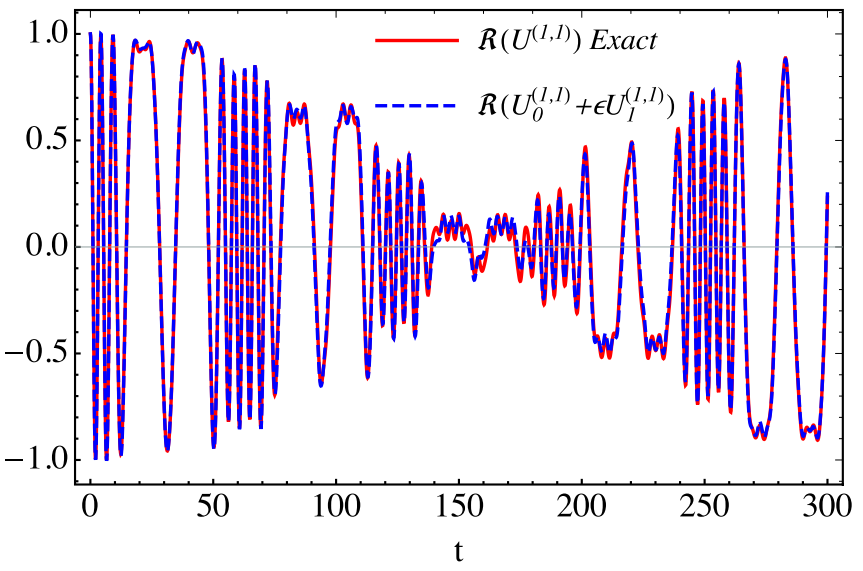

FIG. 3. Comparison between the exact and the approximate dynamics to first order in $\epsilon$ for the real part of $U^{1,1}(t)$, in the presence of resonances [Eq. (19)]. The short-time dynamics is well captured by the unperturbed solution, but the slow oscillations at longer times are obtained from the renormalization of resonances. Parameters: $\omega / \tilde{\Delta}_{z}=0.1, \Omega / \tilde{\Delta}_{z}=2, \beta / \tilde{\Delta}_{z}=0.2$, and $\mu / \tilde{\Delta}_{z}=2$. In this case the dominant resonance is obtained for $n= \pm 10$, and its contribution perfectly captures the slow modulation of the oscillations.

\section{CONCLUSIONS}

We have demonstrated that bichromatic driving provides new possibilities to externally control quantum systems. An interesting one is that two high frequencies $\omega_{1,2}$ can produce effective adiabatic evolution, with frequency controlled by the difference $\omega_{1}-\omega_{2}$. This effect requires strongly driving the system beyond the perturbative regime, and provides an example of the breakdown of high-frequency expansions.

The effect can be used in experiments where low frequencies are out of reach due to equipment restrictions, or if the slow, monochromatic drive resonantly couples to environmental (or undesired) degrees of freedom [41]. This is because the effective Hamiltonian [Eq. (6)] is a function of the coupling strength between the drive and each degree of freedom (in this case controlled by Bessel functions). Then, as the coupling to the environmental modes is different, their Fourier components will be tuned at a different rate with the field amplitude and generally suppressed, while the one of interest is being enhanced. This would allow reaching the desired monochromatic behavior for the degree of freedom of interest, while reducing the undesired signal from the environment. ${ }^{1}$

As the effective adiabatic Hamiltonian [Eq. (8)] generally contains high-frequency corrections, we have also studied their effect. This is equivalent to a bichromatic system with slow and fast frequencies, coupled to different, noncommuting degrees of freedom. In this case, we have shown that the high-frequency corrections of the effective Hamiltonian

\footnotetext{
${ }^{1}$ As a final comment, notice that electronic systems coupled via the Peierls phase to the AC source directly lead to Hamiltonians such as Eq. (4). This means that the transformation to the interaction picture becomes unnecessary and our analysis directly applies.
}

can be used to engineer controlled single-qubit rotations. At short timescales the adiabatic part dominates, and one can switch between free and adiabatic evolution by adjusting the frequency difference $\omega_{-}=\omega_{1}-\omega_{2}$. At longer timescales the high-frequency corrections become relevant and produce adiabatic evolution between the ground state and the excited state. This extra adiabatic evolution along a perpendicular direction is controlled by resonances involving the slow and the fast frequencies, and its period depends on the amplitude of both time-dependent terms $(\mu / \omega$ and $\beta)$. This provides a highly tunable mechanism to implement single-qubit gates using two off-resonant fields only.

Further applications of our results are the possibility to externally control quantum pumping $[42,43]$ in higher dimensional systems [44], or to describe Floquet topological phases at low frequencies. This is because our approach (multiple-scales analysis) allows a complete characterization of the evolution operator, which is required for the topological analysis [45]. In qubits, it would also be interesting to study the competition between geometric and dynamical phases, as the difference between frequencies is tuned. This could be implemented in several experimental setups such as quantum dots, nitrogen-vacancy centers, single-ion magnets, or superconducting junctions.

\section{ACKNOWLEDGMENTS}

This work was supported by the Spanish Ministry of Economy and Competitiveness through Grant No. MAT201786717-P and we acknowledge support from CSIC Research Platform PTI-001. Á.G.-L. acknowledges the Juan de la Cierva program.

\section{APPENDIX A: EFFECTIVE ADIABATIC HAMILTONIAN FOR THE BICHROMATIC TWO-LEVEL SYSTEM}

The Hamiltonian for the two-level system driven by multichromatic driving is given by

$$
\begin{gathered}
H(t)=H_{0}+V(t), \\
H_{0}=\frac{\Delta_{z}}{2} \sigma_{z}, \\
V(t)=\sum_{i} \frac{V_{i}}{2} \cos \left(\omega_{i} t+\phi_{i}\right) \sigma_{x} .
\end{gathered}
$$

The non-perturbative expression in the field amplitudes is obtained from the transformation to the interaction picture:

$$
\begin{gathered}
\tilde{H}(t)=\mathcal{U}(t)^{\dagger} H(t) \mathcal{U}(t)-i \mathcal{U}(t)^{\dagger} \dot{\mathcal{U}}(t), \\
\mathcal{U}(t)=\exp \left\{-i \int V(t) d t\right\} .
\end{gathered}
$$

Simplifying the expressions, the general form can be written as

$$
\begin{aligned}
\tilde{H}(t)= & \frac{\Delta_{z}}{2} \cos \left[\sum_{i} F_{i}\left(t, \omega_{i}, \phi_{i}\right)\right] \sigma_{z} \\
& +\frac{\Delta_{z}}{2} \sin \left[\sum_{i} F_{i}\left(t, \omega_{i}, \phi_{i}\right)\right] \sigma_{y}
\end{aligned}
$$




$$
\begin{aligned}
= & \frac{\Delta_{z}}{4}\left(\sigma_{z}-i \sigma_{y}\right) \prod_{i} e^{i F_{i}\left(t, \omega_{i}, \phi_{i}\right)} \\
& +\frac{\Delta_{z}}{4}\left(\sigma_{z}+i \sigma_{y}\right) \prod_{i} e^{-i F_{i}\left(t, \omega_{i}, \phi_{i}\right)} .
\end{aligned}
$$

Considering the bichromatic case, we find that the transformed Hamiltonian reduces to $\left(\alpha_{i}=V_{i} / \omega_{i}\right)$ :

$$
\begin{aligned}
\tilde{H}(t)= & \frac{\Delta_{z}}{2} \cos \left[\alpha_{1} \sin \left(\omega_{1} t+\phi_{1}\right)+\alpha_{2} \sin \left(\omega_{2} t+\phi_{2}\right)\right] \sigma_{z} \\
& +\frac{\Delta_{z}}{2} \sin \left[\alpha_{1} \sin \left(\omega_{1} t+\phi_{1}\right)\right. \\
& \left.+\alpha_{2} \sin \left(\omega_{2} t+\phi_{2}\right)\right] \sigma_{y} .
\end{aligned}
$$

Using the Jacobi-Anger expansion in terms of Bessel functions, we find the Fourier components

$$
\begin{aligned}
\tilde{H}_{n_{1}, n_{2}}= & \int_{0}^{2 \pi} \frac{d \theta_{1}}{2 \pi} \int_{0}^{2 \pi} \frac{d \theta_{2}}{2 \pi} \tilde{H}\left(\theta_{1}, \theta_{2}\right) e^{-i\left(n_{1} \theta_{1}+n_{2} \theta_{2}\right)}, \text { (A9) } \\
\tilde{H}\left(\theta_{1}, \theta_{2}\right)= & \frac{\Delta_{z}}{2} \cos \left[\alpha_{1} \sin \left(\theta_{1}+\phi_{1}\right)+\alpha_{2} \sin \left(\theta_{2}+\phi_{2}\right)\right] \sigma_{z} \\
& +\frac{\Delta_{z}}{2} \sin \left[\alpha_{1} \sin \left(\theta_{1}+\phi_{1}\right)\right. \\
& \left.+\alpha_{2} \sin \left(\theta_{2}+\phi_{2}\right)\right] \sigma_{y}, \\
\tilde{H}_{n_{1}, n_{2}}= & \frac{\Delta_{z}}{4} e^{i\left(n_{1} \phi_{1}+n_{2} \phi_{2}\right)} J_{n_{1}}\left(\alpha_{1}\right) J_{n_{2}}\left(\alpha_{2}\right)\left(\sigma_{z}-i \sigma_{y}\right) \\
& +\frac{\Delta_{z}}{4} e^{i\left(n_{1} \phi_{1}+n_{2} \phi_{2}\right)} J_{-n_{1}}\left(\alpha_{1}\right) J_{-n_{2}}\left(\alpha_{2}\right)\left(\sigma_{z}+i \sigma_{y}\right)
\end{aligned}
$$

where we have parametrized $\theta_{i}=\omega_{i} t$. To find an effective adiabatic time evolution, we are interested in maximizing the Fourier components which contain the frequency differences $\tilde{H}_{ \pm 1, \mp 1}$, while suppressing the others. If we choose the first maximum of $J_{1}\left(\alpha_{i}\right)$, the Hamiltonian is given by $\left(\omega_{ \pm}=\right.$ $\omega_{1} \pm \omega_{2}$ and we choose the two fields in phase for simplicity $\left.\phi_{1,2}=0\right)$ :

$$
\begin{aligned}
\tilde{H}(t) \simeq & \frac{\Delta_{z}}{2} J_{0}^{2}(\alpha) \sigma_{z}-\Delta_{z} J_{1}^{2}(\alpha)\left[\cos \left(\omega_{-} t\right)-\cos \left(\omega_{+} t\right)\right] \sigma_{z} \\
& +\Delta_{z} J_{0}(\alpha) J_{1}(\alpha) \sum_{i=1,2} \sin \left(\omega_{i} t\right) \sigma_{y}+\cdots,
\end{aligned}
$$

and its dominant contribution consists of

$$
\tilde{H}_{0}(t) \simeq \Delta_{z}\left[\frac{J_{0}^{2}(\alpha)}{2}-J_{1}^{2}(\alpha) \cos \left(\omega_{-} t\right)\right] \sigma_{z}
$$

This is obtained by noticing that the high-frequency terms approximately average to zero.

In general, the effective Hamiltonian contains three types of corrections:

(1) Constant terms such as $\Delta_{z} J_{0}^{2}(\alpha) \sigma_{z} / 2$. They introduce a linear phase evolution for the states (see Fig. 4, blue).

(2) High-frequency corrections which commute with $\tilde{H}(t)$, such as $J_{1}^{2}(\alpha) \cos \left(\omega_{+} t\right) \sigma_{z}$. They introduce fast oscillating nonlinear corrections to the phase evolution.

(3) Noncommuting, time-dependent terms, such as $J_{0}(\alpha) J_{1}(\alpha) \sin \left(\omega_{i} t\right) \sigma_{y}$. They produce transitions between the ground state and the excited state, and can lead to resonances.

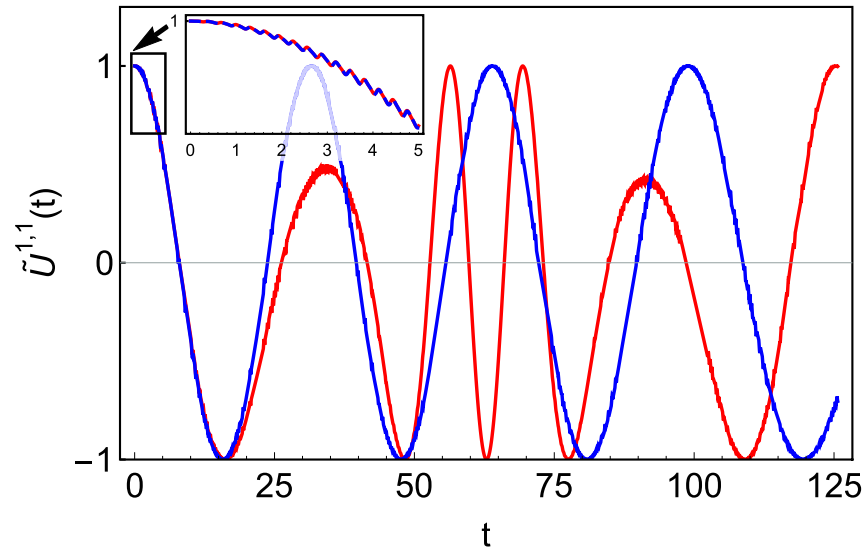

FIG. 4. In red (blue), the dynamics upper diagonal component of the time evolution operator for $\omega_{1} / \Delta_{z}=10, \omega_{2} / \Delta_{z}=10.05(10.01)$, $\alpha_{i} \simeq 1.8$ [this is the first maximum of $J_{1}\left(\alpha_{i}\right)$ ] and $\phi_{i}=0$. The inset shows the effect of the high-frequency corrections, as small oscillations with the frequency $\omega_{i}$ of the original drive. For $\omega_{-}=0.01$ (blue) the static part of the effective Hamiltonian dominates at short time, displaying harmonic oscillations with nonlinearities taking over at later times (not shown). For $\omega_{-}=0.05$ (red) the nonlinear part produces an earlier adiabatic frequency modulation.

Nevertheless, if each independent frequency is large enough, the averaged Hamiltonian in Eq. (A13) is a good approximation, and one is left with the static and the frequency difference $\omega_{-}$terms only, representing the adiabatic evolution. Figure 4 shows an exact numerical simulation of the time-evolution operator using the exact Hamiltonian in Eq. (A6). Notice how the time evolution is adiabatic, with frequency controlled by $\omega_{-}$, and just with small high-frequency oscillations around the mean value due to the extra harmonics. Importantly, as the individual frequencies $\omega_{i}$ are large, compared with the amplitudes of the extra harmonics, corrections coming from high-frequency terms are strongly suppressed.

In conclusion, if the individual frequencies $\omega_{i}$ are large, the dynamics is controlled by just two contributions: (i) the static part, which controls the simple harmonic oscillations (blue in Fig. 4), and (ii) the nonlinear adiabatic term (red in Fig. 4). Furthermore, the ratio between these two could be independently controlled with an extra DC field, which could enhance or suppress the static part.

\section{APPENDIX B: MULTIPLE-SCALES ANALYSIS FOR THE BICHROMATIC CASE}

We consider the general time-dependent Hamiltonian

$$
\begin{aligned}
& \tilde{H}(t)=\tilde{H}_{0}(t)+\epsilon \tilde{H}_{1}(t) \\
& =\frac{1}{2} \tilde{\Delta}(t) \sigma_{z}+\frac{1}{2} \epsilon \tilde{V}(t) \sigma_{y},
\end{aligned}
$$

where $\epsilon$ is a dimensionless parameter which is used to organize the perturbative series, and it is taken to 1 at the end of the calculations. The time-evolution operator obeys the following equation of motion:

$$
i \partial_{t} U(t)=\tilde{H}(t) U(t) .
$$




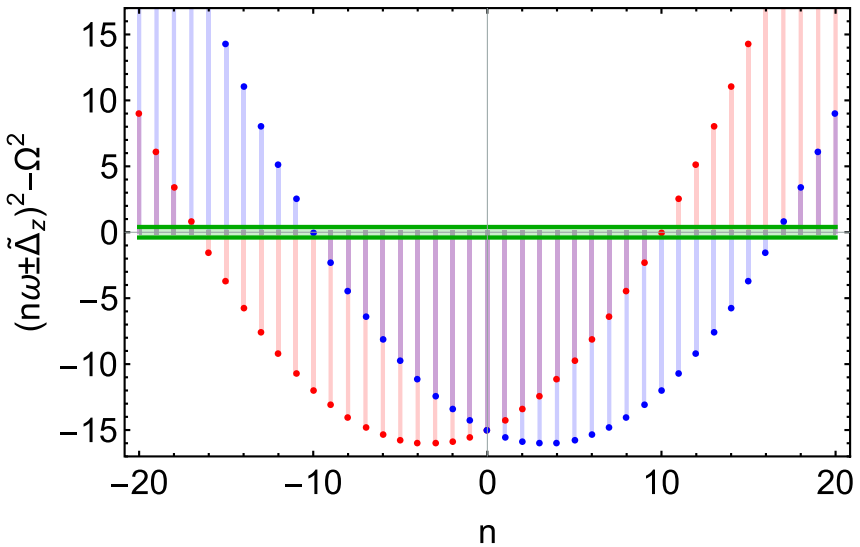

FIG. 5. Curves $\left(n \omega \pm \tilde{\Delta}_{z}\right)^{2}-\Omega^{2}$ for different values of $n$ (red and blue dots) and a region of width $\beta$ [green, see Eq. (B10)]. Points in the green area fulfill the resonance condition, and require renormalization. Increasing $\omega$ from adiabatic to diabatic values produces a transition from several resonances to none. Parameters for the plot: $\Omega / \tilde{\Delta}_{z}=4, \omega / \tilde{\Delta}_{z}=0.3$, and $\beta / \tilde{\Delta}_{z}=0.4$.

Now we write this equation in the usual form of multiplescales analysis, with parametrization $\tau_{n}=\epsilon^{n} t$. The unperturbed solution is obtained from the unperturbed equation of motion:

$$
i \partial_{\tau_{0}} U_{0}(\vec{\tau})=\tilde{H}_{0}\left(\tau_{0}\right) U_{0}(\vec{\tau}),
$$

which gives

$$
U_{0}(\vec{\tau})=e^{-i \int_{0}^{\tau_{0}} \tilde{H}_{0}\left(\tau_{0}\right) d \tau_{0}} u_{0}\left(\tau_{1}\right) .
$$

To first order in $\epsilon$, the equation of motion is given by

$$
i \partial_{\tau_{0}} U_{1}(\vec{\tau})+i \partial_{\tau_{1}} U_{0}(\vec{\tau})=\tilde{H}_{0}\left(\tau_{0}\right) U_{1}(\vec{\tau})+\tilde{H}_{1}\left(\tau_{0}\right) U_{0}(\vec{\tau}),
$$

It can be solved by choosing $U_{1}(\vec{\tau})=u_{1}(\vec{\tau}) v_{1}(\vec{\tau})$, with $u_{1}(\vec{\tau})=e^{-i \int_{0}^{\tau_{0}} \tilde{H}_{0}\left(\tau_{0}\right) d \tau_{0}}$ being the solution to the homogeneous equation. Then, one finds that the solution is given by

$$
\begin{aligned}
U_{1}(\vec{\tau})= & -i u_{1}\left(\tau_{0}\right) \int_{0}^{\tau_{0}} \tilde{H}_{1}^{\prime}\left(\tau_{0}\right) d \tau_{0} u_{0}\left(\tau_{1}\right) \\
& -\tau_{0} u_{1}\left(\tau_{0}\right) \partial_{\tau_{1}} u_{0}\left(\tau_{1}\right),
\end{aligned}
$$

$$
\tilde{H}_{1}^{\prime}\left(\tau_{0}\right)=e^{i \int_{0}^{\tau_{0}} \tilde{H}_{0}\left(\tau_{0}\right) d \tau_{0}} \tilde{H}_{1}\left(\tau_{0}\right) e^{-i \int_{0}^{\tau_{0}} \tilde{H}_{0}\left(\tau_{0}\right) d \tau_{0}} .
$$

Now we choose specific forms for $\tilde{\Delta}(t)$ and $\tilde{V}(t)$ :

$$
\begin{gathered}
\tilde{\Delta}(t)=\tilde{\Delta}_{z}+\mu \cos (\omega t), \\
\tilde{V}(t)=\beta \cos (\Omega t),
\end{gathered}
$$

where $\tilde{\Delta}_{z}$ corresponds to the static part of the effective Hamiltonian [first term in Eq. (A13)], $\mu$ to the dominant periodic modulation with frequency $\omega$ [second term in Eq. (A13)], and $\beta$ to the transverse oscillating correction with frequency $\Omega$. This way, the unperturbed solution corresponds to

$$
U_{0}(\vec{\tau})=e^{-\frac{i}{2}\left[\tilde{\Delta}_{z} \tau_{0}+\frac{\mu}{\omega} \sin \left(\omega \tau_{0}\right)\right] \sigma_{z}} u_{0}\left(\tau_{1}\right)
$$

and the first-order solution is obtained from the rotated Hamiltonian:

$$
\begin{aligned}
\tilde{H}_{1}^{\prime}\left(\tau_{0}\right)= & -i e^{\frac{i}{2}\left[\tilde{\Delta}_{z} \tau_{0}+\frac{\mu}{\omega} \sin \left(\omega \tau_{0}\right)\right] \sigma_{z}} \tilde{H}_{1}\left(\tau_{0}\right) e^{-\frac{i}{2}\left[\tilde{\Delta}_{z} \tau_{0}+\frac{\mu}{\omega} \sin \left(\omega \tau_{0}\right)\right] \sigma_{z}} \\
= & \frac{i}{2} \beta \cos \left(\Omega \tau_{0}\right) \\
& \times\left(\begin{array}{cc}
0 & -e^{-i\left[\tilde{\Delta}_{z} \tau_{0}+\frac{\mu}{\omega} \sin \left(\omega \tau_{0}\right)\right]} \\
e^{i\left[\tilde{\Delta}_{z} \tau_{0}+\frac{\mu}{\omega} \sin \left(\omega \tau_{0}\right)\right]} & 0
\end{array}\right) .
\end{aligned}
$$

To calculate the correction from Eq. (B7), one can write the exponentials in terms of Bessel functions,

$$
\begin{aligned}
\tilde{H}_{1}^{\prime}\left(\tau_{0}\right)= & \frac{i}{2} \beta \cos \left(\Omega \tau_{0}\right) \sum_{n} e^{i n \omega \tau_{0}} \\
& \times\left(\begin{array}{cc}
0 & -e^{-i \tilde{\Delta}_{z} \tau_{0}} J_{-n}\left(\frac{\mu}{\omega}\right) \\
e^{i \tilde{\Delta}_{z} \tau_{0}} J_{n}\left(\frac{\mu}{\omega}\right) & 0
\end{array}\right),
\end{aligned}
$$

and perform the following integrals:

$$
\int_{0}^{\tau_{0}} e^{i\left(n \omega \pm \tilde{\Delta}_{z} \pm \Omega\right) \tau_{0}} d \tau_{0}=i \frac{1-e^{i\left(n \omega \pm \tilde{\Delta}_{z} \pm \Omega\right) \tau_{0}}}{n \omega \pm \tilde{\Delta}_{z} \pm \Omega} .
$$

These integrals will produce secular terms if $\tilde{\Delta}_{z} \pm n \omega \mp \Omega=$ 0 , but they can be canceled by the term $\partial_{\tau_{1}} u_{0}\left(\tau_{1}\right)$ in Eq. (B7),

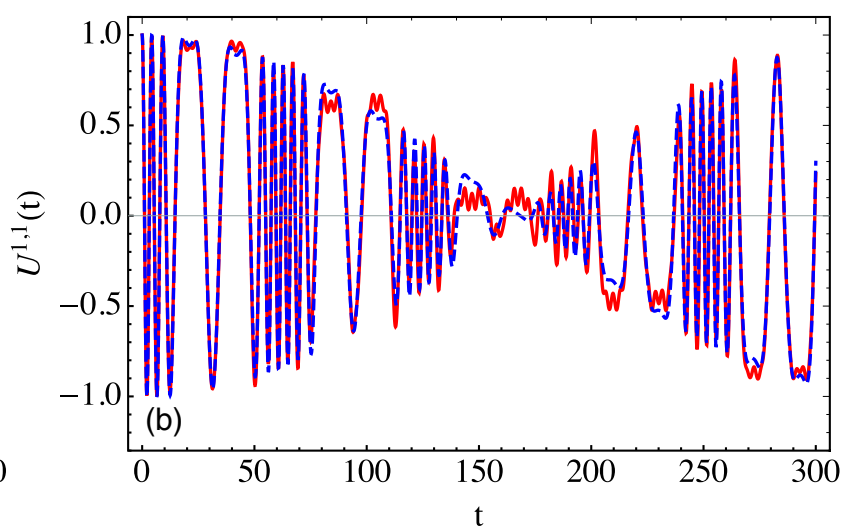

FIG. 6. Comparison between the exact dynamics (red solid) and the one generated by $U_{0}(t)$ (blue dashed). (a) shows the case without renormalization, while (b) shows the case with renormalization of the resonances. We have plotted the real part of the $U^{1,1}(t)$ component, but the agreement is valid for all the other components as well. The addition of small corrections of order $\beta$ to the renormalized $U_{0}(t)$ leads to an even better agreement, as shown in Fig. 3 of the main text. Parameters: $\omega / \tilde{\Delta}_{z}=0.1, \Omega / \tilde{\Delta}_{z}=2, \beta / \tilde{\Delta}_{z}=0.2$, and $\mu / \tilde{\Delta}_{z}=2$. 


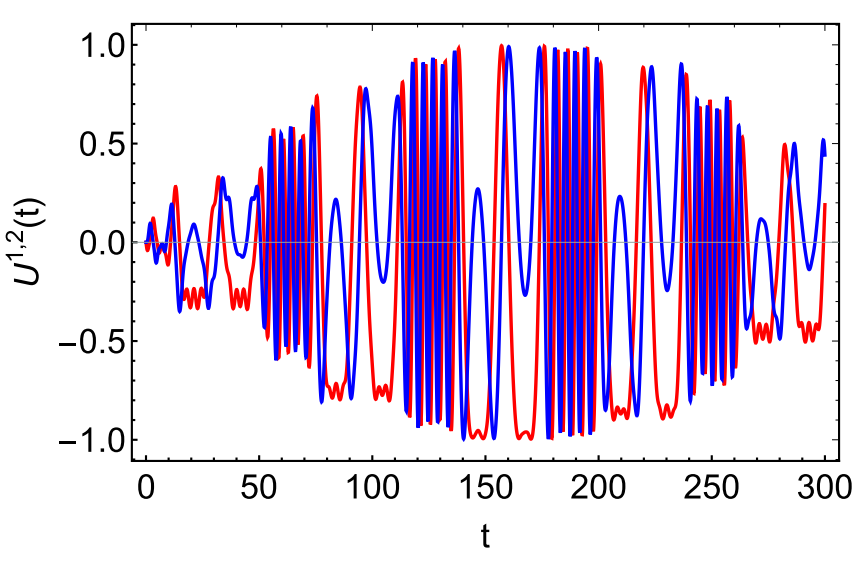

FIG. 7. Real and imaginary parts (red and blue, respectively) of $U^{1,2}(t)$ for the parameters $\omega / \tilde{\Delta}_{z}=0.1, \Omega / \tilde{\Delta}_{z}=2, \beta / \tilde{\Delta}_{z}=0.2$, and $\mu / \tilde{\Delta}_{z}=2$. One can see that the off-diagonal element acquires nonperturbative corrections (of order 1), as predicted by the renormalization of resonances.

defining the flow equation. Furthermore, even for case where the resonance condition is approximately fulfilled $\tilde{\Delta}_{z} \pm n \omega \mp$ $\Omega \lesssim \beta$ only, the perturbative solution would not converge, and the renormalization can be applied. Fig. 5 graphically shows, for a specific case, the harmonics $n$ that require renormalization $(n=10$ in this case). Larger $\omega$ increases the distance between different harmonics, making more difficult to find a resonance. In this case, beyond $\omega \gtrsim 5$ resonances are no longer possible, signaling the transition to the high-frequency regime. $^{2}$ Once the subset of $n$ values which produces secular terms is identified, the cancellation with $\partial_{\tau_{1}} u_{0}\left(\tau_{1}\right)$ in Eq. (B7) produces the following flow equation:

$$
\partial_{\tau_{1}} u_{0}\left(\tau_{1}\right)=-i \frac{\beta}{4} \sum_{n_{0}} J_{n_{0}}\left(\frac{\mu}{\omega}\right) \sigma_{y} u_{0}\left(\tau_{1}\right),
$$

where $n_{0}$ is the set of pairs of integers fulfilling the condition $n_{0} \simeq \pm \frac{\Omega \pm \tilde{\Delta}_{z}}{\omega}$. Then, the lowest order solution is given by

$$
U_{0}(t) \simeq e^{-\frac{i}{2} t\left[\tilde{\Delta}_{z}+\frac{\mu}{\omega} \sin (\omega t)\right] \sigma_{z}} e^{-i t \frac{\beta}{4} \sum_{n_{0}} J_{n_{0}}\left(\frac{\mu}{\omega}\right) \sigma_{y}} .
$$

This non-perturbative correction indicates that the oscillations of each independent energy level are now modulated by a transition between the ground and the excited level with frequency $\frac{\beta}{4} \sum_{n_{0}} J_{n_{0}}\left(\frac{\mu}{\omega}\right)$. Figure 6 compares the exact dynamics with the one generated by $U_{0}(t)$ without and with renormalization (left and right, respectively). The addition of nonsecular contributions produces the plot in the main text (Fig. 3). However, the importance of the resonances is evident from the comparison between the left and right plots for $U_{0}(t)$ in Fig. 6. Finally, we plot in Fig. 7 the exact dynamics of $U^{1,2}(t)$, to confirm that the resonances control the rotation proportional to $\sigma_{y}$ and that their contribution is nonperturbative. This is confirmed by noticing that the off-diagonal part initially vanishes, but acquires values of the order of 1 for times of approximately half the period obtained from the resonances.

\footnotetext{
${ }^{2}$ This is not in contradiction with the first part of the paper, where two high frequencies can give rise to adiabatic evolution. In that case the system is far from the perturbative regime.
}

[1] N. H. Lindner, G. Refael, and V. Galitski, Floquet topological insulator in semiconductor quantum wells, Nat. Phys. 7, 490 (2011).

[2] A. G. Grushin, A. Gómez-León, and T. Neupert, Floquet Fractional Chern Insulators, Phys. Rev. Lett. 112, 156801 (2014).

[3] D. V. Else, B. Bauer, and C. Nayak, Floquet Time Crystals, Phys. Rev. Lett. 117, 090402 (2016).

[4] D. Yudin, D. R. Gulevich, and M. Titov, Light-Induced Anisotropic Skyrmion and Stripe Phases in a Rashba Ferromagnet, Phys. Rev. Lett. 119, 147202 (2017).

[5] A. Gómez-León and P. C. E. Stamp, Dynamical quantum phase transitions in presence of a spin bath, Phys. Rev. B 95, 054402 (2017).

[6] G. Engelhardt, M. Benito, G. Platero, and T. Brandes, Topological Instabilities in ac-Driven Bosonic Systems, Phys. Rev. Lett. 117, 045302 (2016).

[7] J. R. Petta, A. C. Johnson, J. M. Taylor, E. A. Laird, A. Yacoby, M. D. Lukin et al., Coherent manipulation of coupled electron spins in semiconductor quantum dots, Science 309, 2180 (2005)

[8] A. Gómez-León and G. Platero, Charge localization and dynamical spin locking in double quantum dots driven by ac magnetic fields, Phys. Rev. B 84, 121310 (2011).
[9] A. Gómez-León and G. Platero, Transport blocking and topological phases using ac magnetic fields, Phys. Rev. B 85, 245319 (2012).

[10] R. Sánchez and G. Platero, Dark Bell states in tunnel-coupled spin qubits, Phys. Rev. B 87, 081305 (2013).

[11] J. Picó-Cortés, F. Gallego-Marcos, and G. Platero, Direct transfer of two-electron quantum states in ac-driven triple quantum dots, Phys. Rev. B 99, 155421 (2019).

[12] A. Gómez-León and G. Platero, Floquet-Bloch Theory and Topology in Periodically Driven Lattices, Phys. Rev. Lett. 110, 200403 (2013).

[13] M. S. Rudner, N. H. Lindner, E. Berg, and M. Levin, Anomalous Edge States and the Bulk-Edge Correspondence for Periodically Driven Two-Dimensional Systems, Phys. Rev. X 3, 031005 (2013).

[14] P. Delplace, A. Gómez-León, and G. Platero, Merging of Dirac points and Floquet topological transitions in ac-driven graphene, Phys. Rev. B 88, 245422 (2013).

[15] A. Gómez-León, P. Delplace, and G. Platero, Engineering anomalous quantum Hall plateaus and antichiral states with ac fields, Phys. Rev. B 89, 205408 (2014).

[16] M. Benito, A. Gómez-León, V. M. Bastidas, T. Brandes, and G. Platero, Floquet engineering of long-range $p$-wave superconductivity, Phys. Rev. B 90, 205127 (2014). 
[17] T. Kwapiński, S. Kohler, and P. Hänggi, Discontinuous conductance of bichromatically ac-gated quantum wires, Phys. Rev. B. 79, 155315 (2009).

[18] A. A. Abdumalikov, Jr., J. M. Fink, K. Juliusson, M. Pechal, S. Berger, A. Wallraff et al., Experimental realization of nonAbelian non-adiabatic geometric gates, Nature (London) 496, 482 (2013).

[19] F. Forster, M. Mühlbacher, R. Blattmann, D. Schuh, W. Wegscheider, S. Ludwig et al., Landau-Zener interference at bichromatic driving, Phys. Rev. B 92, 245422 (2015).

[20] I. Martin, G. Refael, and B. Halperin, Topological Frequency Conversion in Strongly Driven Quantum Systems, Phys. Rev. X 7, 041008 (2017).

[21] T. H. Stievater, X. Li, D. G. Steel, D. Gammon, D. S. Katzer, D. Park et al., Rabi Oscillations of Excitons in Single Quantum Dots, Phys. Rev. Lett. 87, 133603 (2001).

[22] H. Kamada, H. Gotoh, J. Temmyo, T. Takagahara, and H. Ando, Exciton Rabi Oscillation in a Single Quantum Dot, Phys. Rev. Lett. 87, 246401 (2001).

[23] H. Htoon, T. Takagahara, D. Kulik, O. Baklenov, A. L. Holmes, and C. K. Shih, Interplay of Rabi Oscillations and Quantum Interference in Semiconductor Quantum Dots, Phys. Rev. Lett. 88, 087401 (2002).

[24] F. H. L. Koppens, C. Buizert, K. J. Tielrooij, I. T. Vink, K. C. Nowack, T. Meunier et al., Driven coherent oscillations of a single electron spin in a quantum dot, Nature (London) $\mathbf{4 4 2}$, 766 (2006).

[25] A. Morello, O. N. Bakharev, H. B. Brom, R. Sessoli, and L. J. de Jongh, Nuclear Spin Dynamics in the Quantum Regime of a Single-Molecule Magnet, Phys. Rev. Lett. 93, 197202 (2004).

[26] C. Schlegel, J. van Slageren, M. Manoli, E. K. Brechin, and M. Dressel, Direct Observation of Quantum Coherence in SingleMolecule Magnets, Phys. Rev. Lett. 101, 147203 (2008).

[27] Y. Nakamura, Y. A. Pashkin, and J. S. Tsai, Rabi Oscillations in a Josephson-Junction Charge Two-Level System, Phys. Rev. Lett. 87, 246601 (2001).

[28] G. Platero and R. Aguado, Photon-assisted transport in semiconductor nanostructures, Phys. Rep. 395, 1 (2004).

[29] R. Kubo, Statistical-mechanical theory of irreversible processes. I. general theory and simple applications to magnetic and conduction problems, J. Phys. Soc. Jpn. 12, 570 (1957).
[30] S. Blanes, F. Casas, J. A. Oteo, and J. Ros, The Magnus expansion and some of its applications, Phys. Rep. 470, 151 (2009).

[31] B. Pérez-González, M. Bello, G. Platero, and A. Gómez-León, Simulation of 1D Topological Phases in Driven Quantum Dot Arrays, Phys. Rev. Lett. 123, 126401 (2019).

[32] A. Díaz-Fernández, E. Díaz, A. Gómez-León, G. Platero, and F. Domínguez-Adame, Floquet engineering of Dirac cones on the surface of a topological insulator, Phys. Rev. B 100, 075412 (2019).

[33] V. S. Malinovsky and J. L. Krause, General theory of population transfer by adiabatic rapid passage with intense, chirped laser pulses, Eur. Phys. J. D 14, 147 (2001).

[34] F. Nathan and M. S. Rudner, Topological singularities and the general classification of Floquet-Bloch systems, New J. Phys. 17, 125014 (2015).

[35] M. Grifoni and P. Hanggi, Driven quantum tunneling, Phys. Rep. 304, 229 (1998).

[36] T. S. Ho, S. I. Chu, and J. V. Tietz, Semiclassical many-mode floquet theory, Chem. Phys. Lett. 96, 464 (1983).

[37] M. Abramowitz and I. A. Stegun, Handbook of Mathematical Functions with Formulas, Graphs, and Mathematical Tables (Dover, Mineola, NY, 1972).

[38] J. Kevorkian and J. D. Cole, Multiple Scale and Singular Perturbation Methods (Springer, Berlin, 1996).

[39] M. Janowicz, Method of multiple scales in quantum optics, Phys. Rep. 375, 327 (2003).

[40] A. Gómez-León, Spin bath dynamics and dynamical renormalization group, Phys. Rev. B 100, 094308 (2019).

[41] J. A. Jones, V. Vedral, A. Ekert, and G. Castagnoli, Geometric quantum computation using nuclear magnetic resonance, Nature (London) 403, 869 (2000).

[42] D. J. Thouless, Quantization of particle transport, Phys. Rev. B 27, 6083 (1983).

[43] S. Nakajima, T. Tomita, S. Taie, T. Ichinose, H. Ozawa, L. Wang et al., Topological Thouless pumping of ultracold fermions, Nat. Phys. 12, 296 (2016).

[44]

[45] S. Yao, Z. Yan, and Z. Wang, Topological invariants of Floquet systems: General formulation, special properties, and Floquet topological defects, Phys. Rev. B 96, 195303 (2017). 